\title{
ON WAVE PROPAGATION IN A DEFORMED MOONEY-RIVLIN MATERIAL*
}

\author{
BY M. HAYWS (Lniversity College, Dublin)
}

Summary. For a homogeneously deformed incompressible Mooney-Rivlin material it is shown that there is a universal connection between the speeds of propagation of the plane transverse waves which may propagate in any direction in the matcrial. Such connections are not valid, in general, for incompressible materials.

1. Introduction. According to the classical linear elasticity theory of materials which are not internally constrained, it is well known that three plane waves may propagate in any direction $\mathbf{n}$ in the material. If the phase speeds of the three plane waves propagation in the direction $\mathbf{n}$ are denoted by $c_{\alpha}(\mathbf{n}), \alpha=1,2,3$ it has been shown [1, 2] that there is a universal connection

$$
\sum_{\alpha=1}^{3}\left\{c_{\alpha}{ }^{2}(\mathbf{n})+{c_{\alpha}}^{2}(\mathbf{m})+{c_{\alpha}}^{2}(\mathbf{p})\right\}=\sum_{\alpha=1}^{3}\left\{c_{\alpha}{ }^{2}(\mathbf{r})+{c_{\alpha}}^{2}(\mathbf{s})+c_{\alpha}{ }^{2}(\mathrm{t})\right\}
$$

relating the wave speeds. Here $(\mathbf{n}, \mathbf{m}, \mathbf{p})$ and $(\mathbf{r}, \mathbf{s}, \mathbf{t})$ are any two orthogonal triads of directions. It was pointed out in [2] that connections of this sort do not hold in general in materials which are subject to internal constraints such as incompressibility, or inextensibility in a particular direction. This is due to the fact that the acoustical tensor involves $\mathbf{n}$ in a quadratic way for unconstrained materials, whereas if the material is incompressible, for example, the acoustical tensor involves $\mathbf{n}$ in a quartic fashion.

The purpose of this note is to point out that for a particular incompressible isotropic elastic material, the Mooney-Rivlin model, a universal connection of the form of (1.1) does exist between the wave speeds in a specimen of the material when it has been subjected to a pure homogeneous deformation. Furthermore, this universal connection involves directly the strain-energy density of the material in the state of homogencous deformation.

2. Universal connections. When the generic particle initially at $\mathrm{X}$ in a virgin Mooney-Rivlin material is displaced to $\mathbf{x}$, the strain-energy per unit volume is $W$, given by

$$
2 W=C(I-3)+D(I I-3)
$$

where $C$ and $D$ are constants and**

$$
I=B_{i i}, 2 I I=I^{2}-B_{i j} B_{i i} .
$$

* Received December 8,1975 .

** Unless stated to the contrary, repeated suffixes are summed over 1, 2, 3. All equations are referred to Cartesian coordinates $x_{i}$. 
$B_{i j}$ are the components in the rectangular Cartesian coordinate system $x$ of the left Cauchy-(ireen strain tensor given by

$$
B_{i j}=\left(\partial x_{i} / \partial X_{A}\right)\left(\partial x_{i} / \partial X_{A}\right) .
$$

Since the material is incompressible, $\operatorname{det} \mathbf{B}=1$.

When the material is in a virgin state it is subjected to a pure static homogeneous deformation $x_{\alpha}=\lambda_{\alpha} X_{\alpha}\left(\alpha=1,2,3\right.$; no sum) with $\lambda_{1} \lambda_{2} \lambda_{3}=1$. Superposed on this is an infinitesimal time-dependent deformation $\bar{x}_{i}=x_{i}+\epsilon u_{i}\left(x_{i}, t\right)$, where $\epsilon$ is assumed so small that squares and higher powers of $\epsilon$ may be neglected in comparison with firstdegree terms. In view of the incompressibility constraint,

$$
\partial u_{i} / \partial x_{i}=0 .
$$

If

$$
u_{i}=a_{i} \exp \iota\left(n_{i} x_{j}-c t\right), \quad a_{i} n_{i}=0, \quad a_{i} a_{i}=n_{i} n_{i}=1,
$$

this represents a plane transverse wave polarized along $\mathbf{a}$, propagating in the direction n with speed $c$.

It has been shown [3] that two transverse plane waves may propagate in any direction $\mathbf{n}$ in the deformed material. The amplitudes of these waves are at right angles to each other and to $\mathbf{n}$. They are the proper vectors, other than $\mathbf{n}$, of $\boldsymbol{\Gamma}(\mathbf{n})$ where

$$
\Gamma_{k m}(\mathbf{n})=\widetilde{B}_{k q} n_{q} \widetilde{B}_{i m} n_{i}-\widetilde{B}_{i j} n_{i} n_{i} \widetilde{B}_{k m} .
$$

Here $\tilde{\mathbf{B}}$ is the strain tensor corresponding to the static homogeneous deformation and is given by

$$
\widetilde{B}_{i j}=\lambda_{1}{ }^{2} \delta_{i 1} \delta_{i 1}+\lambda_{2}{ }^{2} \delta_{i 2} \delta_{i 2}+\lambda_{3}{ }^{2} \delta_{i 3} \delta_{j 3},
$$

with corresponding invariants $\tilde{I}, \tilde{I} I$ given by

$$
\tilde{I}=\lambda_{1}{ }^{2}+\lambda_{2}{ }^{2}+\lambda_{3}{ }^{2}, \quad \tilde{I} \tilde{I}=\lambda_{1}{ }^{2} \lambda_{2}{ }^{2}+\lambda_{2}{ }^{2} \lambda_{3}{ }^{2}+\lambda_{3}{ }^{2} \lambda_{1}{ }^{2} .
$$

Furthermore, the speed $c(\mathbf{n} ; \mathbf{a})$ of the wave propagating in the direction $\mathbf{n}$ with unit amplitude $\mathbf{a}$ is given [3] by

$$
\begin{aligned}
\rho c^{2}(\mathbf{n} ; \mathbf{a})= & (C+\widetilde{I} D) \widetilde{B}_{i j} n_{i} n_{i}-D \widetilde{B}_{i j} \widetilde{B}_{i k} n_{i} n_{k} \\
& +D n_{i} \widetilde{B}_{i m} a_{m} n_{k} \widetilde{B}_{k q} a_{q} \\
& -D \widetilde{B}_{r s} n_{r} n_{s} \widetilde{B}_{k m} a_{k} a_{m},
\end{aligned}
$$

where $\rho$ is the constant density of the material. Thus if $b$ is the unit amplitude of the other wave propagating in the direction $\mathbf{n}$, then it follows that

$$
\rho c^{2}(\mathbf{n} ; \mathbf{a})+\rho c^{2}(\mathbf{n} ; \mathbf{b})=2 C \widetilde{B}_{i j} n_{i} n_{i}+D\left\{\tilde{I} \widetilde{B}_{i j} n_{i} n_{i}-\widetilde{B}_{i j} \widetilde{B}_{j k} n_{i} n_{k}\right\},
$$

since $\mathbf{n}, \mathbf{a}$ and $\mathbf{b}$ form an orthogonal triad of unit vectors.

If, for brevity, the phase speeds of the two waves propagating along $\mathbf{n}$ are denoted by $c_{\alpha}(\mathbf{n}), \alpha=1,2$, then it follows from (2.10) for the orthogonal triad of directions $(\mathbf{n}, \mathbf{m}, \mathbf{p})$ that

$$
\rho \sum_{\alpha=1}^{2}\left\{c_{\alpha}{ }^{2}(\mathbf{n})+c_{\alpha}{ }^{2}(\mathbf{m})+c_{\alpha}{ }^{2}(\mathbf{p})\right\}=2 C \tilde{I}+2 D \tilde{I} \tilde{I} .
$$


The right side of this equation is independent of $\mathbf{n}, \mathbf{m}$ and $\mathbf{p}$. Thus for any two orthogonal triads $(\mathbf{n}, \mathbf{m}, \mathbf{p})$ and $\mathbf{r}, \mathbf{s}, \mathbf{t})$ there is the universal connection

$$
\sum_{\alpha=1}^{2}\left\{c_{\alpha}{ }^{2}(\mathbf{n})+c_{\alpha}{ }^{2}(\mathbf{m})+c_{\alpha}{ }^{2}(\mathbf{p})\right\}=\sum_{\alpha=1}^{2}\left\{c_{\alpha}{ }^{2}(\mathbf{r})+c_{\alpha}{ }^{2}(\mathbf{s})+c_{\alpha}{ }^{2}(\mathbf{t})\right\} .
$$

A corollary of (2.12) is that if $\mathbf{n}, \mathbf{m}, \mathbf{r}$ and $\mathbf{s}$ are any coplanar unit vectors with $\mathbf{n} \cdot \mathbf{m}$ $=\mathbf{r} \cdot \mathbf{s}=0$ then

$$
\sum_{\alpha=1}^{2}\left\{c^{2}(\mathrm{n})+c^{2}(\mathrm{~m})\right\}=\sum_{\alpha=1}^{2}\left\{c^{2}(\mathbf{r})+c^{2}(\mathbf{s})\right\} .
$$

By use of a counterexample it was shown in [2] that such universal connections do not hold in general for incompressible materials. Thus the Mooney-Rivlin material is special in this respect.

Suppose now that two identical virgin specimens of Mooney-Rivlin material are subjected to two different homogeneous deformations $\left(\lambda_{1}, \lambda_{2}, \lambda_{3}\right)$ and $\left(\bar{\lambda}_{1}, \bar{\lambda}_{2}, \bar{\lambda}_{3}\right)$ (say), in an obvious notation. The axes of the deformation are common. Let the phase speeds in the direction $\mathbf{n}$ in the two materials be denoted by $c_{\alpha}(\mathbf{n})$ and $\bar{c}_{\alpha}(\mathbf{n}), \alpha=1,2$. Then from (2.1) and (2.11) there follows the universal connection involving the two different basic homogeneous deformations:

$$
\begin{aligned}
4\{W(\boldsymbol{\lambda})- & W(\bar{\lambda})\} \\
& =\rho \sum_{\alpha=1}^{2}\left\{\left(c_{\alpha}{ }^{2}(\mathrm{n})-\bar{c}_{\alpha}{ }^{2}(\mathbf{n})\right)+\left(c_{\alpha}{ }^{2}(\mathrm{~m})-\bar{c}_{\alpha}{ }^{2}(\mathrm{~m})\right)+\left(c_{\alpha}{ }^{2}(\mathbf{p})-\bar{c}_{\alpha}{ }^{2}(\mathbf{p})\right)\right\},
\end{aligned}
$$

where $W(\lambda)$ and $W(\bar{\lambda})$ are the strain-energies per unit volume for the two bodies.

Of course in the particular case when $\lambda_{1}=\lambda_{2}=\lambda_{3}=1$, all the transverse waves travel with the same speed $c_{0}$ (say) given by

$$
\rho c_{n}^{2}=(C+D) \text {. }
$$

Thus a particular case of (2.14) gives

$$
4 W(\lambda)=\rho \sum_{\alpha=1}^{2}\left\{c_{\alpha}{ }^{2}(\mathbf{n})+c_{\alpha}{ }^{2}(\mathbf{m})+c_{\alpha}{ }^{2}(\mathbf{p})\right\}-6 \rho c_{0}{ }^{2} .
$$

3. Concluding remarks. It has been pointed out in [2] that the main value of universal connections of this sort is that they provide a check on his results for the experimentalist.

Currie and Hayes [3] showed that for homogeneously deformed Mooney-Rivlin materials there is no essential difference between the manner of propagation of infinitesmal and finite-amplitude plane waves. For any direction $\mathbf{n}$ in the deformed material, two finite-amplitude transverse plane waves may propagate without change of shape, and their speed of propagation is the same as the speed of the corresponding infinitesimal waves. Thus in the universal connections obtained here it is immaterial whether infinitesimal plane waves or finite-amplitude plane waves are considered.

\section{RefERENCES}

[1] E. R. Fitzgerald and T. W. Wright, Phys. Stat. Sol. 24, 37-44 (1967)

[2] M. Hayes, Arch. Rat. Mech. Anal. 46, 105-1143 (1972)

[3] P. Currie and M. Hayes, J. Inst. Maths. Applics. 5, 140-161 (1969) 